\title{
Acquisition of the Conjugative Virulence Plasmid From a CG23 Hypervirulent Klebsiella pneumoniae Strain Enhances Bacterial Virulence
}

OPEN ACCESS

Edited by: Yang Zhang,

University of Pennsylvania,

United States

Reviewed by:

Hao Zhang,

University of Pennsylvania,

United States

Yu Zhou,

Institut Pasteur of Shanghai (CAS),

China

Wenen Liu,

Central South University, China

*Correspondence:

Xiaofei Jiang

jiangxi2154@sina.com

Zike Sheng

shengz12@fudan.edu.cn

Yunkun Huang

hykhn@sina.com

${ }^{\dagger}$ These authors have contributed equally to this work

Specialty section:

This article was submitted to Clinical Microbiology,

a section of the journal

Frontiers in Cellular and

Infection Microbiology

Received: 02 August 2021

Accepted: 25 August 2021

Published: 09 September 2021

Citation:

Tian D, Wang W, Li M, Chen W, Zhou $Y$, Huang $Y$, Sheng $Z$ and Jiang $X$ (2021) Acquisition of the Conjugative

Virulence Plasmid From a CG23 Hypervirulent Klebsiella pneumoniae Strain Enhances Bacterial Virulence. Front. Cell. Infect. Microbiol. 11:752011.

doi: $10.3389 /$ fcimb.2021.752011

\author{
Dongxing Tian ${ }^{1 \dagger}$, Weiwen Wang ${ }^{1 \dagger}$, Meng $\mathrm{Li}^{2+}$, Wenjie Chen ${ }^{3}$, Ying Zhou ${ }^{4}$, \\ Yunkun Huang ${ }^{5^{*}}$, Zike Sheng ${ }^{6 *}$ and Xiaofei Jiang ${ }^{1 *}$
}

\begin{abstract}
1 Department of Clinical Laboratory, Huashan Hospital of Fudan University, Shanghai, China, 2 Department of Clinical Laboratory, The First Affiliated Hospital of Guangxi Medical University, Nanning, Guangxi, China, ${ }^{3}$ Department of Infectious Disease, Huashan Hospital of Fudan University, Shanghai, China, ${ }^{4}$ Department of Clinical Laboratory, Shanghai Pulmonary Hospital of Tongji University, Shanghai, China, ${ }^{5}$ Department of Clinical Laboratory, Kunming Yan'an Hospital, Kunming, China, ${ }^{6}$ Department of Infectious Diseases, Ruijin Hospital, Shanghai Jiao Tong University School of Medicine, Shanghai, China
\end{abstract}

The emergence of hypervirulent and carbapenem-resistant Klebsiella pneumoniae (hv-CRKP) has become a hot topic and confounding problem for clinicians and researchers alike. Conjugative virulence plasmids have the potential to cause more threatening dissemination of hv-CRKP strains. We previously identified K2606, a CG23 clinical hypervirulent strain of Klebsiella pneumoniae harboring a conjugative virulence plasmid designated pK2606. In this study we examined hypervirulence levels using assays of biofilm formation, serum resistance, and wax larvae and mouse in vivo infection models. Moreover, to define the transfer ability of pK2606 and whether this confers hypervirulence to other strains we performed plasmid transconjugation experiments between K2606 and the ST11 CRKP strain HS11286 along with E. coli J53. We found that although biofilm formation and serum resistance were not significantly increased, the transconjugants acquired the ability of produce high level of siderophores and also caused high mortality of wax larvae and mice. Furthermore, we identified pK2606-like conjugative virulence plasmids in GenBank, providing evidence that such plasmids may have begun to spread throughout China. These findings provide an evidence base for the possible mechanisms of the emergence of hv-CRKP strains and highlight the potential of pK2606-like conjugative virulence plasmids to spread worldwide.

Keywords: conjugative, transfer, dissemination, plasmid, siderophore production, hypervirulence, hypervirulent Klebsiella pneumoniae

\section{INTRODUCTION}

Klebsiella pneumoniae is considered as a major threat to public health. This microorganism is not only cause severe invasive disease that affect healthy people in the community, but also has a significant propensity to acquire drug resistance in the nosocomial setting (Navon-Venezia et al., 2017; Harada and Doi, 2018). However, while strains rarely possess both of these properties, there are increasing reports of multidrug-resistant and hypervirulent strains (Wyres et al., 2019; Zhang et al., 2020). Traditionally, hypervirulent strains have only been related with some particular types, 
such as the capsular type K1 sequence type 23 (K1-ST23) and K2-ST86 (Bialek-Davenet et al., 2014; Lam et al., 2018a), which are commonly susceptible to antibiotics. More recently, virulence clusters have also been identified in other STs which are always associated with resistance, such as ST11 and ST15 (Gu et al., 2018; Shu et al., 2019). These virulence clusters, including iron acquisition regulatory genes (especially aerobactin, but also salmochelin) and regulators of mucoid phenotype A ( $r m p A)$ and $\operatorname{rmp} A 2$, are carried on a large virulence plasmid.

The emergence of hypervirulent and carbapenem-resistant Klebsiella pneumoniae has become a hot topic, and research is increasingly focused on the mechanism of transmission, but this currently remains unclear. The virulence plasmids acquired by ST11 carbapenem-resistant Klebsiella pneumoniae (CRKP) are commonly pK2044-like plasmids, which are considered nonconjugative (Smillie et al., 2010; Tian et al., 2021). According to several studies these virulence plasmids confer hypervirulence to other strains ( $\mathrm{Gu}$ et al., 2018; Zhang et al., 2020). Although uncommon, virulence plasmids with complete Type IV Secretion Systems (T4SSs) must also not be overlooked. Conjugative virulence plasmids have the potential to transfer to other resistant strains or species, endowing them with a hypervirulent and resistant phenotype. Due to their self-conjugation ability, the encoded critical virulence factors of conjugative virulence plasmids, especially the aerobactin genes iucABCD-iutA, will be transferred along with the plasmid. This combination of hypervirulence and resistance poses a serious challenge to both clinicians and researchers. Indeed, once conjugative virulence plasmids are transferred among different strains in the hospital setting, the situation becomes even more intractable.

Our earlier study provided a scheme to distinguish conjugative virulence plasmids from non-conjugative virulence plasmids by assessment of IncFIB $_{\mathrm{K}}$ genetic diversity (Tian et al., 2021). However, the virulence level conferred by conjugative virulence plasmids needs to be further characterized. CG23 is considered to be highly related to conserved hvKp strains that harbor pK2044-like virulence plasmids (Lam et al., 2018b), whereas conjugative virulence plasmids are possibly associated with other clonal groups. We recently identified a CG23 clinical strain K2606 from a patient with bloodstream infections. K2606 harbored a conjugative virulence plasmid, while no pK2044-like virulence plasmid was found in it. Nevertheless, the virulence of the K2606 strain and transfer ability of pK2606 plasmid need to be determined. In particular, it also remains urgent to confirm whether such conjugative virulence plasmids confer hypervirulence to other strains.

\section{MATERIALS AND METHODS}

\section{Strains and Growth Conditions}

K. pneumoniae strain K2606 was isolated from blood in 2012 at Huashan Hospital (Shanghai, China), and were sequenced completely (GCA_011006575.1). Carbapenem-resistant Klebsiella pneumoniae HS11286 was isolated from sputum in 2011 at Huashan Hospital (Shanghai, China) and were sequenced completely before
(GCA_000240185.2) (Liu et al., 2012). All strains were stored in $20 \%(\mathrm{w} / \mathrm{v})$ glycerol at $-80^{\circ} \mathrm{C}$ and cultured on Luria Broth medium at $37^{\circ} \mathrm{C}$. Plasmid stability experiments were performed as described previously (Zhou et al., 2020). For LB growth experiments, the bacteria was grown at a starting $\mathrm{A}_{600}$ of 0.05 in $\mathrm{LB}$ at $37^{\circ} \mathrm{C}$, and the $\mathrm{A}_{600}$ was measured each hour. Each curve was performed in triplicate.

\section{Antimicrobial Susceptibility Test}

The antimicrobial susceptibility was performed using the microdilution method and interpreted according to the Clinical and Laboratory Standards Institute (CLSI, 2020). Antimicrobial agents tested included amikacin, ampicillin/ sulbactam, piperacillin/tazobactam, cefazolin, cefuroxime, ceftriaxone, cefepime, chloramphenicol, gentamicin, levofloxacin, trimethoprim/sulphamethoxazole, meropenem, tigecycline, and nitrofurantion.

\section{Conjugation Assay}

Carbapenem-resistant K. pneumoniae HS11286 and sodium azide-resistant $E$. coli $\mathrm{J} 53$ were used as a recipient, and $K$. pneumoniae K2606 strain was used as the donor. The kanamycin-resistant gene was introduced into pK2606 by $\lambda$-Red-mediated homologous recombination method to be selected as the donor resistance marker. The primers were included in Table S3. Both donors and recipients were cultured to logarithmic phase at $37^{\circ} \mathrm{C}$, and $200 \mathrm{ul}$ of donor cells and $800 \mathrm{ul}$ of recipient cells were mixed and inoculated on the LB agar plate at $37^{\circ} \mathrm{C}$ overnight. Next, the transconjugants were selected with the meropenem (1ug/ml) and hygromycin (400ug/ml) if HS11286 was used as a recipient, or sodium azide $(150 \mathrm{ug} / \mathrm{ml})$ and hygromycin $(400 \mathrm{ug} / \mathrm{ml})$ if E. coli J53 was used as a recipient. The transconjugants was determined by PCR using iucA, oq $x A$, and ICE as marker genes. PFGE, S1-PFGE, and plasmids draft sequencing were performed as described previously (Pecora et al., 2015; Tian et al., 2020). Sequences were submitted to GenBank (MZ475693, MZ475694, MZ475695, MZ475696, MZ475699, MZ475705, MZ475706, MZ475707, and MZ475708).

\section{Biofilm Formation Assay}

Biofilm formation was performed as described previously in 96well microtiter plates (Choi and Ko, 2015). Briefly, 200 $\mu$ l of the mid-log phase bacteria cells $\left(1.5 \times 10^{7} \mathrm{CFU} / \mathrm{ml}\right)$ was added to 96 well microtiter plates and incubated overnight. Next, all cultures were removed and then washed twice with phosphate-buffered saline. $0.1 \%$ crystal violet solution was used for staining for 20 min and washed three times. The biofilm formation was quantified by measuring the $\mathrm{A}_{590}$ after solubilized with $200 \mu \mathrm{l}$ of $95 \%$ ethanol. Each assay was performed in duplicate and repeated three times independently.

\section{Serum Resistance Assay}

The serum resistance was determined according to a previous study (Choi and Ko, 2015). The mid-log phase bacteria cells were mixed with normal human serum at a $1: 3$ ratios and then incubated at $37^{\circ} \mathrm{C}$ for $2 \mathrm{~h}$. Serum resistance were characterized by plotting the average survival percentage of each strain against 
the incubation time. All assays were performed three times independently. K. pneumoniae strains were considered resistant to normal human serum if at least $90 \%$ of the organisms survived and were considered sensitive to serum if CFU counts dropped to $1 \%$ after $2 \mathrm{~h}$ of incubation.

\section{Greater Wax Moth Larvae and Mouse Infection Models}

G. mellonella larvae weighing about $300 \mathrm{mg}$, which were purchased from the Tianjin Huiyude Biotech Company, were used to determine the virulence level of the strains. The tested strains were adjusted with PBS $\left(1 \times 10^{6} \mathrm{CFU} / \mathrm{ml}\right)$. A group of ten G. mellonella larvae was infected with $10 \mu \mathrm{l}$ of PBS and other groups were injected with $10 \mu \mathrm{l}$ of bacteria cultures, and incubated at $37^{\circ} \mathrm{C}$ for $72 \mathrm{~h}$ to observe the survival of them.

Male BALB/c mice (average approximately 16g, 5 weeks old) were purchased from the Shanghai Medical Laboratory Animal Center (Shanghai, China). Eight mice in each group were infected intravenously with $1 \times 10^{7} \mathrm{CFU}$ bacteria. The mortality rate was recorded for $72 \mathrm{~h}$. Animal experiments were repeated twice. Survival curves were generated by Prism 8 . Animal ethics approval was obtained from Animal Ethics Committee of Huashan Hospital of Fudan University.

\section{Siderophores Production Experiment}

The relative quantitative siderophores in chelated M9 minimal medium (c-M9) was determined as described previously (Payne, 1994). Briefly, the siderophore assay solution was made by $50 \mathrm{ml}$ of $1.2 \mathrm{mM}$ hexadecyltrimethylammonium bromide, $1.5 \mathrm{ml}$ of 1 $\mathrm{mM} \mathrm{FeCl}_{3} \cdot 6 \mathrm{H}_{2} \mathrm{O}, 7.5 \mathrm{ml}$ of $2 \mathrm{mM}$ chrome azurol S (CAS), and
1.37 M piperazine ( $\mathrm{pH}=5.6) .100 \mathrm{ul}$ of each sample was added to wells of a flat-bottom 96-well plate, and then $100 \mathrm{ul}$ of siderophores assay solution with $2 \% \quad 0.2 \mathrm{M}$ 5-sulfosalicyclic acid was added. The reaction mixture was incubated for $30 \mathrm{~min}$, and were measured at A680 nm. The c-M9 was plus siderophores assay solution plus 5-sulfosalicyclic acid solution was used as a reference (r). The sample (s) should have a lower absorbance than the reference. Siderophore units $(\mathrm{Su})$ are defined as [( $\mathrm{Ar}-\mathrm{As}) / \mathrm{Ar}] \times$ $100=\%$ siderophore units. Each assay was performed in duplicate and repeated three times independently.

Siderophore production was also detected by the CAS agar plate assay (Schwyn and Neilands, 1987). The stationary-phase iron-cheated cultures $(3 \mu \mathrm{l})$ were dropped on CAS plates, and siderophore production was determined by the orange halos after incubation for $48 \mathrm{~h}$ at $37^{\circ} \mathrm{C}$.

\section{Statistical Analysis}

Prism 8 (GraphPad Software, San Diego, CA) was used to calculate the significance with: unpaired, two-tailed, Student's $t$-tests, log-rank test; and two-tailed, Mann-Whitney U-test. The type and number of replicates are indicated in the Figure legends.

\section{RESULTS}

\section{The Conjugative Transfer of pK2606-Like Plasmid Encoding Aerobactin Genes}

K2606 is an ST1027 Klebsiella pneumoniae strain, belonging to clonal group 23 (CG23). It harbors 5 plasmids with sizes of $174647 \mathrm{bp}, 138314 \mathrm{bp}, 109004 \mathrm{bp}, 70729 \mathrm{bp}$, and 10077bp, respectively (Table 1). The pK2606 $\left(\mathrm{IncFIB}_{\mathrm{K}} / \mathrm{IncFII}_{\mathrm{K}}\right)$ plasmid

TABLE 1 | Genomic characteristics of K2606, HS11286, and J53.

\begin{tabular}{|c|c|c|c|c|c|c|c|}
\hline Strain & MLST & $\begin{array}{l}\text { K- } \\
\text { type }\end{array}$ & Chromosome & Plasmids & Resistance determinants & $\begin{array}{c}\text { Virulence } \\
\text { determinants }\end{array}$ & $\begin{array}{c}\text { Molecular } \\
\text { markers }\end{array}$ \\
\hline K2606 & ST1027 & KL20 & $5,313,003$ bp & $\begin{array}{l}\text { unamed1: unidentified, } \\
\text { 174647 bp; } \\
\text { unamed2: IncFIB }{ }_{\mathrm{K}} / \\
\text { IncFII }_{\mathrm{K}}, 138314 \mathrm{bp} ; \\
\text { unamed3: } \\
\text { IncFIB } \mathrm{pKPSH}_{1}, 109004 \mathrm{bp} ; \\
\text { unamed4: IncFI| } \\
\text { 7072911, } \\
\text { unamed5: } \\
\text { CoIRNAI,10077bp; }\end{array}$ & $\begin{array}{l}\text { B-lactam: SHV-27, CTX-M-14; aminoglycoside: aadA16,AAC } \\
\text { (6')-lb-cr6; fosfomycin: FosA5; quinolone: oqxA, QnrB2, } \\
\text { emrR; trimethoprim: dfrA27; sulphonamide: sul1; } \\
\text { tetracycline: tet(A);macrolide: mphA; rifamycin: arr-3. }\end{array}$ & iucABCD-iutA & $\begin{array}{l}\operatorname{oq} \times A(+) \\
\operatorname{ICE}(-)\end{array}$ \\
\hline HS11286 & ST11 & KL103 & $5,333,942$ bp & $\begin{array}{l}\text { pKPSH1: IncFIB }{ }_{\mathrm{pKPSH} 1} \\
122799 \text { bp; } \\
\text { pKPSH2: IncFII } / \text { IncR, } \\
11195 \text { bp; } \\
\text { pKPSH3: IncA/C, } \\
105974 \text { bp; } \\
\text { pKPSH4: Col156, } 3751 \\
\text { bp; } \\
\text { pKPSH5: ColpHAD28, } \\
3353 \text { bp; } \\
\text { pKPSH6: ColpKPSH6, } \\
1308 \text { bp. }\end{array}$ & $\begin{array}{l}\text { } \beta \text {-lactam: KPC-2, SHV-11, CTX-M-14, TEM-1; } \\
\text { aminoglycoside: rmtB, ANT(3'), } \mathrm{AAC}(3), \mathrm{APH}(6), \mathrm{APH}\left(3^{\prime \prime}\right) \text {; } \\
\text { sulphonamide: sul2; tetracycline: tet(D); fosfomycin: FosA6. }\end{array}$ & None & $\begin{array}{l}\operatorname{oq} \times A(-) \\
\operatorname{ICE}(+)\end{array}$ \\
\hline J53 & ST292 & - & 4682574 bp & None & None & None & $\begin{array}{l}\operatorname{oq} \times A(-) \\
\operatorname{ICE}(-)\end{array}$ \\
\hline
\end{tabular}


was found to encode aerobactin genes iucABCD-iutA. HS11286 is an ST11 carbapenem-resistant Klebsiella pneumoniae harboring a KPC plasmid. We found that pK2606 could be conjugated to Klebsiella pneumoniae HS11286 as well as E. coli J53, and the transconjugant strains HS11286-vir2-pK2606 and J53-vir2-pK2606 were successfully obtained with conjugation frequencies of $2.3 \times 10^{-5} \pm 8.9 \times 10^{-5}$ and $6.4 \times 10^{-5} \pm 1.2 \times 10^{-6}$, respectively. PFGE confirmed the genetic relationship between HS11286 and HS11286-vir2-pK2606, and between J53 and J53-vir2-pK2606 (Figure 1). S1-PFGE and draft genome sequencing determined that pK2606 had been transferred to HS11286 and J53 (Figures 1 and S1). Notably, pK2606 was transferred to E. coli J53 together with pK2606-1 (Figure 1). The KPC plasmid pKPHS2 of Klebsiella pneumoniae HS11286 was also self-conjugative. However, we could not obtain the transconjugant of pKPHS2 plasmid transferred to K2606 strain. The E. coli J53 strain was susceptible to all tested antimicrobials, while the K2606 strain was susceptible to amikacin, piperacillin/tazobactam, cefepime, chloramphenicol, gentamicin, meropenem, and tigecycline (Table S1). Notably, the HS11286 strain was resistant to all tested antimicrobials except tigecycline (Table S1).

\section{The Virulence Phenotype of K2606 and Transconjugant Strains}

Although the K2606 strain harbored the pK2606 plasmid encoding aerobactin, its virulence level still needed to be confirmed. The K2606 strain had a lower biofilm formation ability than hypervirulent NTUH-K2044 (Figure 2). Serum resistance experiments showed that the K2606 strain hardly survived in normal healthy serum (Figure 2). Although the K2606 strain did not exhibit good biofilm formation capabilities nor serum resistance, it did show a similarly high siderophore production (Figure 2), indicating that the $i u c A B C D$ iutA aerobactin genes exerted their normal function. K2606 strain also showed a significantly higher virulence levels compared to HS11286 and J53 strains in both mice and wax moth larva infection models (Figure 2).

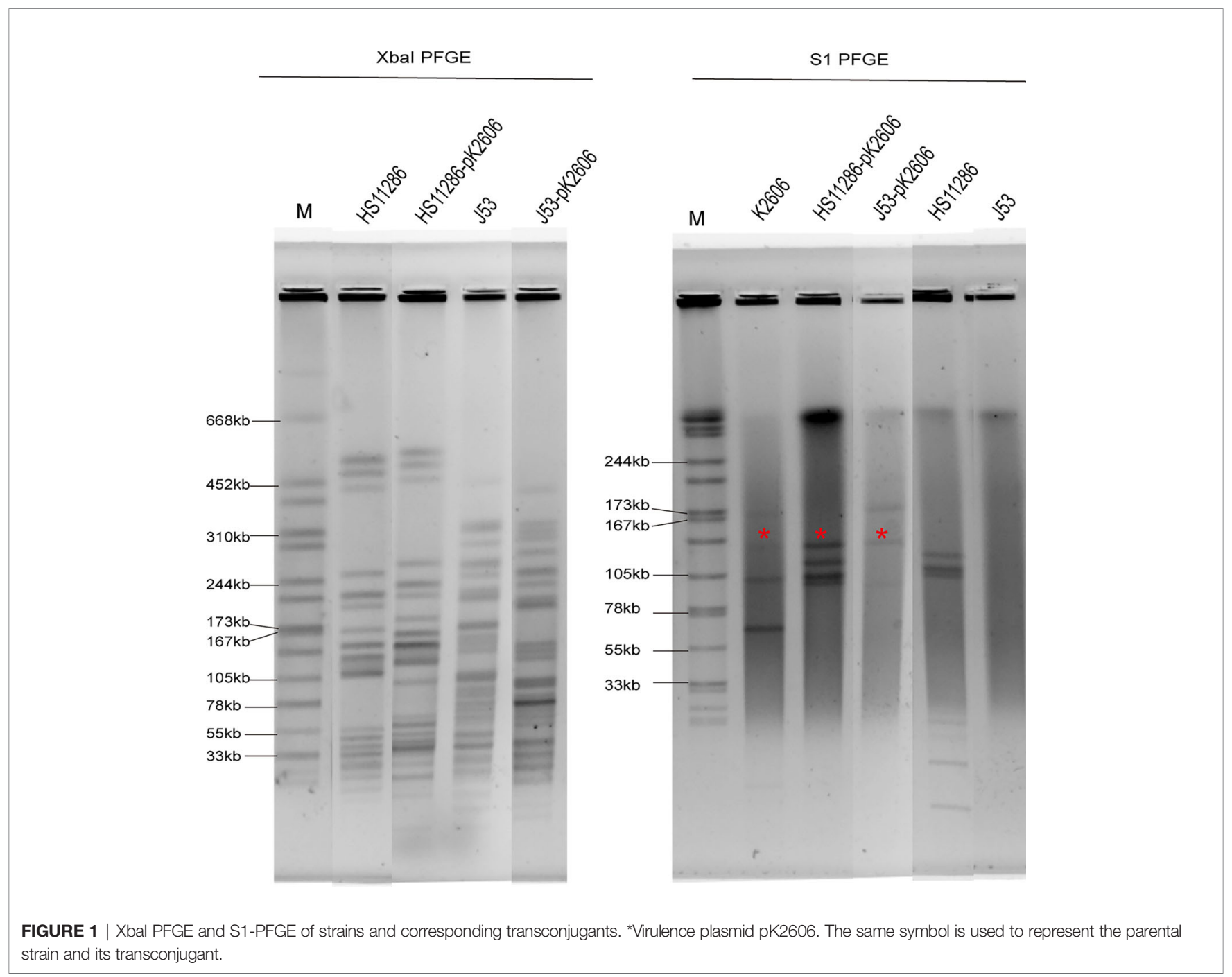


A

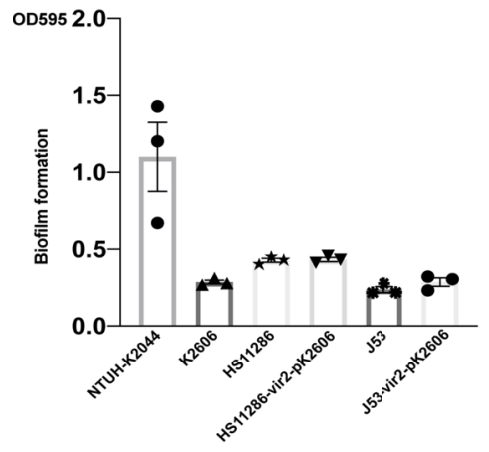

C

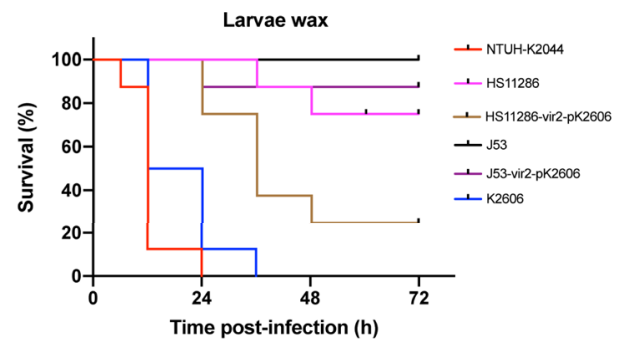

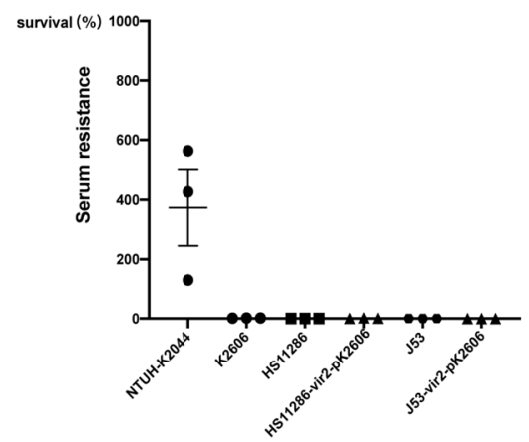

D

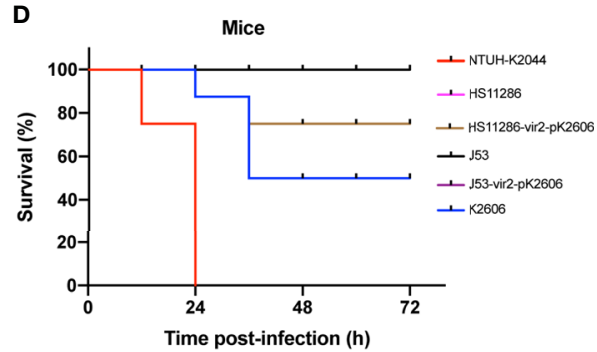

FIGURE 2 | The virulence phenotype of strains and transconjugants. The biofilm formation (A) and serum resistance (B) of K2606, HS11286, J53, HS11286-vir2pK2606, and J53-vir2-pK2606. Hypervirulent strain NTUH-K2044 was used as a positive control. An unpaired two-sided Student's t-test was performed for parental strain and its transconjugant. Each data point was repeated three times $(n=3)$. Data are presented as the mean \pm s.e.m. No significant difference $(P>0.05)$ was observed between HS11286 and HS11286-vir2-pK2606, and between J53 and J53-vir2-pK2606. The virulence level of different strains and their transconjugants as depicted in a larvae wax infection model (C) and a mouse infection model (D). Survival of mice $(n=8)$ infected by each $K$. pneumoniae strain at $72 \mathrm{~h}$ is shown. Hypervirulent NTUH-K2044 was used as a positive control. A log-rank (Mantel-Cox) test was performed for the indicated curves. A significant difference $(P<0.0001$ in both C, D) was observed between HS11286 and HS11286-vir2-pK2606, and between J53 and J53-vir2-pK2606.

Next, we evaluated whether the acquisition of the pK2606 plasmid could enhance virulence of Klebsiella pneumoniae HS11286 and E. coli J53. The pK2606 plasmid was stable in HS1 1286 and J53 after 25 passages. Moreover, the acquisition of pK2606 appeared not to affect the growth rate and antimicrobial susceptibility of HS11286-vir2-pK2606 and J53-vir2-pK2606 compared to their parental strains, indicating a very small fitness cost of pK2606 (Table S1 and Figure S2). The biofilm formation and serum resistance of the transconjugants HS11286vir2-pK2606 and J53-vir2-pK2606 were similar to that of their parental strains (Figures 2A, B). However, the acquisition of pK2606 by HS11286 and J53 caused significantly increased siderophore production, reaching the levels of NTUH-K2044 and K2606 (Figure 3). Moreover, when mice were infected with $1 \times 10^{7} \mathrm{CFU}$ of bacteria, the virulence level of HS11286-vir2pK2606 increased, resulting in $25 \%$ mortality at $72 \mathrm{~h}$, whereas there was $100 \%$ survival using the parental HS11286 strain (Figure 2). The elevated virulence levels were even more evident using the wax larva infection model. Infection of larvae wax with $1 \times 10^{6} \mathrm{CFU}$ of HS11286-vir2-pK2606 led to 25\% survival at $72 \mathrm{~h}$, a level much lower than HS11286 (Figures 2C, D). Notably, the acquisition of pK2606 plasmid by E. coli J53 appeared not to cause increased virulence. To further confirm our observations, we successfully obtained the transconjugant
JS187-vir2-pK2606. The JS187 strain was ST11 carbapenemresistant Klebsiella pneumoniae which was highly similar to HS11286. Our results showed that JS187-vir2-pK2606 acquired the ability of produce high levels of siderophores and caused high mortality of larvae wax and mice (Figures S3 and 3C).

\section{The Prevalence of pK2606-Like Conjugative Virulence Plasmids in Klebsiella pneumoniae}

As shown above, pK2606 plasmid was self-conjugative and conferred high siderophore production to recipient strains, thus increasing their virulence. We collected sixteen pK2606like plasmids from GenBank, which were highly similar to pK2606 and possessed aerobactin genes iucABCD-iutA and the T4SS transfer module (Figure 4). The K2606 strain was ST1027, belonging to CG23, which is commonly associated with hypervirulent Klebsiella pneumoniae strains. Other Klebsiella pneumoniae strains isolated from China in 2019 harboring pK2606-like plasmids were ST1, ST36, ST37, ST437, ST290, and ST967, respectively (Table S2). The ST distribution and diversity of locations in China suggested such conjugative virulence plasmids may have begun to spread throughout China. 
A

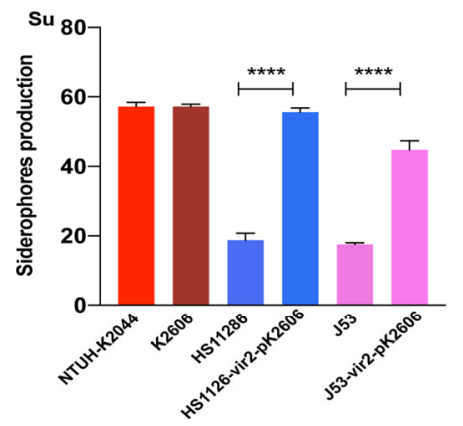

B

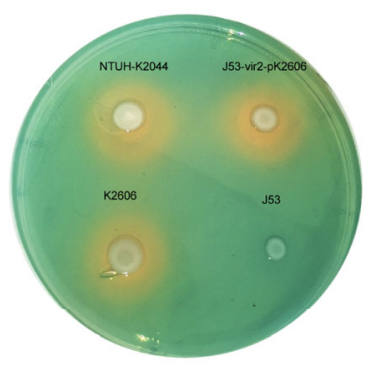

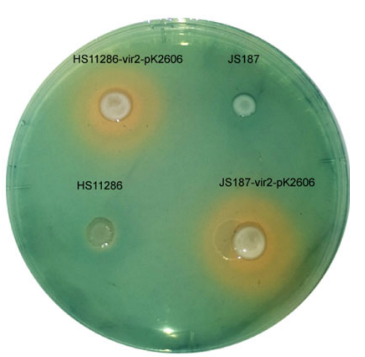

FIGURE 3 | The siderophore production of strains and transconjugants. (A) The quantitative siderophore production of K2606, HS11286, J53, HS11286-vir2pK2606, and J53-vir2-pK2606. An unpaired two-sided Student's t-test was performed for parental strain and its transconjugant. Each data point was repeated three times $(n=3)$. Data are presented as the mean \pm s.e.m. ${ }^{\star \star \star \star} P<0.0001$. (B, C) The CAS agar plate assay indicated the siderophore production. Siderophore production was evaluated by the orange halos.

\section{DISCUSSION}

Two possible evolutionary pathways for hypervirulent and carbapenem-resistant $K$. pneumoniae have been proposed: a) the acquisition of a carbapenem-resistance plasmid by K1/ K2 hvKP strains, known as CR-hvKp (Hennequin and Robin, 2016) and; b) carbapenem-resistant $K$. pneumoniae (CRKP) acquiring a virulence plasmid, recognized as hv-CRKP (Cejas et al., 2014). The most common virulence plasmids acquired by CRKP strains are non-conjugative pK2044-like plasmids, but their mechanism of transfer is still uncertain. Our previous study identified some conjugative virulence plasmids encoding aerobactin genes in Klebsiella pneumoniae (Tian et al., 2021). Considering their potential for self-conjugation, it is essential to confirm the virulence of the host bacteria and their ability to transfer to other strains. CG23 is the most common type of hypervirulent $K$. pneumoniae harboring the pK2044-like virulence plasmid, which contributes to hypervirulence together with its thick capsules (Lam et al., 2018a). We observed a CG23 clinical strain K2606 harboring conjugative

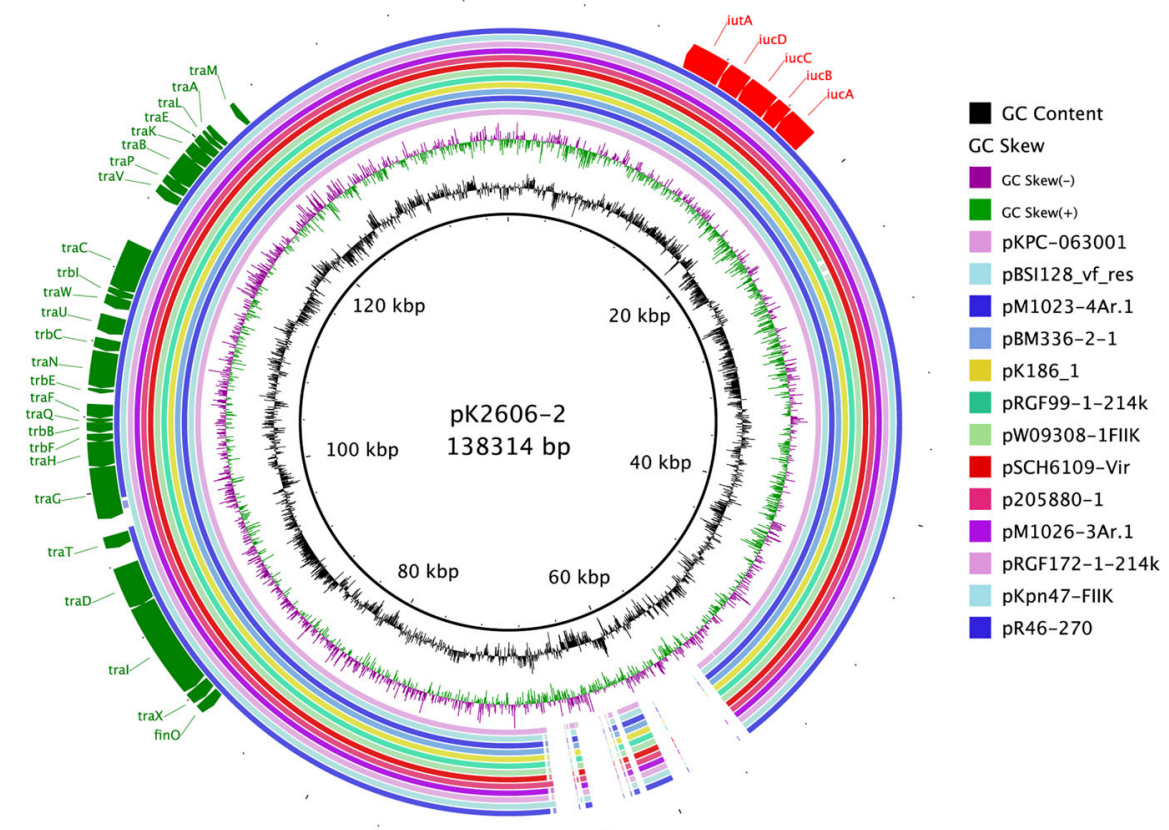

FIGURE 4 | Conjugative pK2606-like virulence plasmids of Klebsiella pneumoniae strains in GenBank. Circular maps were generated using BLAST Ring Image Generator (BRIG). pK2606 was used as a reference. 
plasmid pK2606 encoding aerobactin genes, but no similar pK2044-like plasmids were found. One of the aims of this study was to determine the virulence level of the CG23 K2606 strain and the transfer ability of pK2606.

In comparison to a representative CG23 hypervirulent Klebsiella pneumoniae strain, NTUH-K2044, the K2606 strain was not endowed with good biofilm formation ability nor serum resistance. The K2606 strain lacks thick capsules and polysaccharide production and we speculate that thick capsules may largely contribute to biofilm formation and serum resistance. However, high virulence levels of K2606 were still observed in both mice and larvae wax infection models, indicating that high sideroproduction could also strengthen virulence and facilitate survival in vivo. This does not preclude that there are other virulence factors in the plasmid possibly influencing the virulence as well. However, capsular and siderophore production are always considered as two key factors contributing to the pathogenicity of hvKp strains (Russo and Marr, 2019). Efficient iron acquisition is necessary for hvKp to survive in human ascites. Thus, if the hvKp strain could produce more iron acquisition factors, it may acquire increased virulence. However, determining, whether pK2606 carrying iucABCD-iutA confers virulence to other strains is necessary in order to prevent the dissemination of conjugative pK2606-like virulence plasmids.

Conjugative virulence plasmids were possibly formed by the insertion of an aerobactin cluster into an $\mathrm{IncFIB}_{\mathrm{K}} / \mathrm{IncFII}$ conjugative plasmid (Tian et al., 2021), and the composition of pK2606 is concordant with this observation. There are also some other reports concerning conjugative virulence plasmids. A previous study indicated that the virulence plasmid p15WZ82_Vir with a T4SS in K. variicola could be transferred to a CRKP strain and E. coli (Yang et al., 2019). Li et al. also reported the conjugative virulence plasmid p17-15-vir in Klebsiella pneumoniae ST15 (Li et al., 2020). The two large hybrid virulence plasmids p15WZ-82_Vir $(282 \mathrm{~Kb})$ and p1715 -vir $(479 \mathrm{~Kb})$ were possibly unstable and brought potential fitness costs to their host bacteria. In comparison, pK2606 is just $138 \mathrm{~Kb}$ and possesses a complete Type IV Secretion System with an encoded aerobactin cluster. Notably, pK2606 could efficiently transfer to ST11 CRKP strains and also E. coli J53, conferring high siderophore production abilities and facilitating their survival in vivo. It has long been known that bacteria require iron for growth and that their ability to acquire iron is requisite for growth and survival (Page, 2019). Therefore, most bacteria can produce diverse siderophores, including aerobactin, enterobactin, salmochelin, and yersiniabactin, to acquire iron in iron-depleted environments, such as the human host. Aerobactin is a critical virulence factor for hvKp, while the additional siderophores are not major contributors to high siderophore levels (Russo et al., 2015). The virulence plasmids acquired by ST11 CRKP strains always display deleted salmochelin coding genes, which would not affect their ability to confer hypervirulence to host bacteria ( $\mathrm{Gu}$ et al., 2018). The pK2606 plasmid encodes only aerobactin, and the acquisition of pK2606 by HS11286 and J53 caused significantly increased siderophore production, reaching similarly high levels to the hypervirulent NTUH-K2044. We observed that the transconjugant HS11286-vir2-pK2606 and J53-vir2-pK2606 were not only endowed with high siderophore levels, but also high virulence levels in mice and larvae wax infection models.

Our study confirmed the hypervirulence of the K2606 strain and efficient transfer of the pK2606 plasmid, which could confer virulence to other strains. There are some indications that the conjugative virulence plasmids have been disseminated, for example, pK2606-like conjugative virulence plasmids were identified amongst various STs of Klebsiella pneumoniae strains. CG23 strains do not always carry classical pK2044-like virulence plasmids, and disturbingly, the pK2606-like conjugative virulence plasmids can be successfully transferred to CRKP strains to further evolve into hv-CRKP. Conjugative virulence plasmids almost always carry aerobactin genes, while the mucoid regulator $r m p A$ was not found, thus, may not confer the hypermucoviscous phenotype. However, plasmid-encoded aerobactin appears sufficient to facilitate host bacterial survival and severe infections in vivo (Russo et al., 2015). Nevertheless, the acquisition of pK2606-like conjugative virulence plasmids may not produce as high a virulence phenotype as nonconjugative pK2044-like plasmids, but the consequences of their conjugal transfer are relatively serious, especially transference to multi-drug resistant strains. The deficiency of host immune defense systems in ST11 CRKP strains facilitates the invasion of such conjugative virulence plasmids (Tang et al., 2020; Zhou et al., 2020). Thus, prompt action is needed with specific interventions to prevent another global epidemic like the KPC plasmids。

In conclusion, this study confirmed the hypervirulence phenotype of Klebsiella pneumoniae strain K2606 is caused by high siderophore levels. Additionally, we found that pK2606-like conjugative virulence plasmids could confer virulence to other strains. The pK2606-like virulence plasmids are self-conjugative and have emerged in various Klebsiella pneumoniae strains, including multi-drug resistant strains. Clinicians and researchers should be vigilant for the emergence of hv-CRKP strains caused by the transmission of such conjugative virulence plasmids in the clinical setting.

\section{DATA AVAILABILITY STATEMENT}

The datasets presented in this study can be found in online repositories. The names of the repository/repositories and accession number(s) can be found in the article/Supplementary Material.

\section{ETHICS STATEMENT}

The animal study was reviewed and approved by Animal Ethics Committee of Huashan Hospital of Fudan University. 


\section{AUTHOR CONTRIBUTIONS}

All the authors listed have made a substantial, direct and intellectual contribution to this work, and approved the submitted version for publication.

\section{FUNDING}

This work was supported by Science and Technology Commission of Shanghai Municipality under Grant 19JC1413002, National Natural Science Foundation of China under Grant 81871692 and 82172315, Shanghai Municipal Key Clinical Specialty under grant shslczdzk0330, and Jiaxing

\section{REFERENCES}

Bialek-Davenet, S., Criscuolo, A., Ailloud, F., Passet, V., Jones, L., DelannoyVieillard, A. S., et al. (2014). Genomic Definition of Hypervirulent and Multidrug-Resistant Klebsiella Pneumoniae Clonal Groups. Emerg. Infect. Dis. 20 (11), 1812-1820. doi: 10.3201/eid2011.140206

Cejas, D., Fernández Canigia, L., Rincón Cruz, G., Elena, A. X., Maldonado, I., Gutkind, G. O., et al. (2014). First Isolate of KPC-2-Producing Klebsiella Pneumonaie Sequence Type 23 From the Americas. J. Clin. Microbiol. 52 (9), 3483-3485. doi: 10.1128/JCM.00726-14

Choi, M. J., and Ko, K. S. (2015). Loss of Hypermucoviscosity and Increased Fitness Cost in Colistin-Resistant Klebsiella Pneumoniae Sequence Type 23 Strains. Antimicrob. Agents Chemother. 59 (11), 6763-6773. doi: 10.1128/ AAC.00952-15

CLSI (2020). M100: Performance Standards for Antimicrobial Susceptibility Testing. 30 edition. CLSI Supplement M100. (Wayne, PA: Clinical and Laboratory Standards Institute).

Gu, D., Dong, N., Zheng, Z., Lin, D., Huang, M., Wang, L., et al. (2018). A Fatal Outbreak of ST11 Carbapenem-Resistant Hypervirulent Klebsiella Pneumoniae in a Chinese Hospital: A Molecular Epidemiological Study. Lancet Infect. Dis. 18 (1), 37-46. doi: 10.1016/s1473-3099(17)30489-9

Harada, S., and Doi, Y. (2018). Hypervirulent Klebsiella Pneumoniae: A Call for Consensus Definition and International Collaboration. J. Clin. Microbiol. 56 (9), e00959-18. doi: 10.1128/JCM.00959-18

Hennequin, C., and Robin, F. (2016). Correlation Between Antimicrobial Resistance and Virulence in Klebsiella Pneumoniae. Eur. J. Clin. Microbiol. Infect. Dis. 35 (3), 333-341. doi: 10.1007/s10096-015-2559-7

Lam, M. M. C., Wyres, K. L., Duchene, S., Wick, R. R., Judd, L. M., Gan, Y. H., et al. (2018a). Population Genomics of Hypervirulent Klebsiella Pneumoniae Clonal-Group 23 Reveals Early Emergence and Rapid Global Dissemination. Nat. Commun. 9 (1), 2703. doi: 10.1038/s41467-018-05114-7

Lam, M. M. C., Wyres, K. L., Judd, L. M., Wick, R. R., Jenney, A., Brisse, S., et al. (2018b). Tracking Key Virulence Loci Encoding Aerobactin and Salmochelin Siderophore Synthesis in Klebsiella Pneumoniae. Genome Med. 10 (1), 77. doi: 10.1186/s13073-018-0587-5

Li, R., Cheng, J., Dong, H., Li, L., Liu, W., Zhang, C., et al. (2020). Emergence of a Novel Conjugative Hybrid Virulence Multidrug-Resistant Plasmid in Extensively Drug-Resistant Klebsiella Pneumoniae ST15. Int. J. Antimicrob. Agents 55 (6), 105952. doi: 10.1016/j.ijantimicag.2020.105952

Liu, P., Li, P., Jiang, X., Bi, D., Xie, Y., Tai, C., et al. (2012). Complete Genome Sequence of Klebsiella Pneumoniae Subsp. Pneumoniae HS11286, a Multidrug-Resistant Strain Isolated From Human Sputum. J. Bacteriol 194 (7), 1841-1842. doi: 10.1128/jb.00043-12

Navon-Venezia, S., Kondratyeva, K., and Carattoli, A. (2017). Klebsiella Pneumoniae: A Major Worldwide Source and Shuttle for Antibiotic Resistance. FEMS Microbiol. Rev. 41 (3), 252-275. doi: 10.1093/femsre/fux013

Page, M. G. P. (2019). The Role of Iron and Siderophores in Infection, and the Development of Siderophore Antibiotics. Clin. Infect. Dis. 69 (Suppl 7), S529S537. doi: 10.1093/cid/ciz825
Municipal Key Laboratory of Infectious Diseases and Bacterial Drug Resistance.

\section{ACKNOWLEDGMENTS}

We thank the authority of NTUH-K2044 by Professor Jin-Town Wang from National Taiwan University Hospital.

\section{SUPPLEMENTARY MATERIAL}

The Supplementary Material for this article can be found online at: https://www.frontiersin.org/articles/10.3389/fcimb.2021.752011/ full\#supplementary-material

Payne, S. M. (1994). Detection, Isolation, and Characterization of Siderophores. Methods Enzymol. 235, 329-344. doi: 10.1016/0076-6879(94)35151-1

Pecora, N. D., Li, N., Allard, M., Li, C., Albano, E., Delaney, M., et al. (2015). Genomically Informed Surveillance for Carbapenem-Resistant Enterobacteriaceae in a Health Care System. mBio 6 (4), e01030. doi: 10.1128/ mBio.01030-15

Russo, T. A., and Marr, C. M. (2019). Hypervirulent Klebsiella Pneumoniae. Clin. Microbiol. Rev. 32 (3), e00001-19. doi: 10.1128/cmr.00001-19

Russo, T. A., Olson, R., MacDonald, U., Beanan, J., and Davidson, B. A. (2015). Aerobactin, But Not Yersiniabactin, Salmochelin, or Enterobactin, Enables the Growth/Survival of Hypervirulent (Hypermucoviscous) Klebsiella Pneumoniae Ex Vivo and In Vivo. Infect. Immun. 83 (8), 3325-3333. doi: 10.1128/IAI.00430-15

Schwyn, B., and Neilands, J. B. (1987). Universal Chemical Assay for the Detection and Determination of Siderophores. Anal. Biochem. 160 (1), 47-56. doi: 10.1016/0003-2697(87)90612-9

Shu, L., Dong, N., Lu, J., Zheng, Z., Hu, J., Zeng, W., et al. (2019). Emergence of OXA-232 Carbapenemase-Producing Klebsiella Pneumoniae That Carries a pLVPK-Like Virulence Plasmid Among Elderly Patients in China. Antimicrob. Agents Chemother. 63 (3), e02246-18. doi: 10.1128/AAC.02246-18

Smillie, C., Garcillán-Barcia, M. P., Francia, M. V., Rocha, E. P., and de la Cruz, F. (2010). Mobility of Plasmids. Microbiol. Mol. Biol. Rev. 74 (3), 434-452. doi: $10.1128 / \mathrm{mmbr} .00020-10$

Tang, Y., Fu, P., Zhou, Y., Xie, Y., Jin, J., Wang, B., et al. (2020). Absence of the Type I-E CRISPR-Cas System in Klebsiella Pneumoniae Clonal Complex 258 Is Associated With Dissemination of IncF Epidemic Resistance Plasmids in This Clonal Complex. J. Antimicrob. Chemother. 75 (4), 890-895. doi: 10.1093/jac/ dkz538

Tian, D., Wang, B., Zhang, H., Pan, F., Wang, C., Shi, Y., et al. (2020). Dissemination of the Bla (NDM-5) Gene via IncX3-Type Plasmid Among Enterobacteriaceae in Children. mSphere 5 (1), e00699-19. doi: 10.1128/ mSphere.00699-19

Tian, D., Wang, M., Zhou, Y., Hu, D., Ou, H. Y., and Jiang, X. (2021). Genetic Diversity and Evolution of the Virulence Plasmids Encoding Aerobactin and Salmochelin in Klebsiella Pneumoniae. Virulence 12 (1), 1323-1333. doi: 10.1080/21505594.2021.1924019

Wyres, K. L., Wick, R. R., Judd, L. M., Froumine, R., Tokolyi, A., Gorrie, C. L., et al. (2019). Distinct Evolutionary Dynamics of Horizontal Gene Transfer in Drug Resistant and Virulent Clones of Klebsiella Pneumoniae. PLoS Genet. 15 (4), e1008114. doi: 10.1371/journal.pgen.1008114

Yang, X., Wai-Chi Chan, E., Zhang, R., and Chen, S. (2019). A Conjugative Plasmid That Augments Virulence in Klebsiella Pneumoniae. Nat. Microbiol. 4 (12), 2039-2043. doi: 10.1038/s41564-019-0566-7

Zhang, Y., Jin, L., Ouyang, P., Wang, Q., Wang, R., Wang, J., et al. (2020). Evolution of Hypervirulence in Carbapenem-Resistant Klebsiella Pneumoniae in China: A Multicentre, Molecular Epidemiological Analysis. J. Antimicrob. Chemother. 75 (2), 327-336. doi: 10.1093/jac/dkz446

Zhou, Y., Tang, Y., Fu, P., Tian, D., Yu, L., Huang, Y., et al. (2020). The Type I-E CRISPR-Cas System Influences the Acquisition of blaKPC-IncF Plasmid in 
Klebsiella Pneumonia. Emerg. Microbes Infect. 9 (1), 1011-1022. doi: 10.1080/ 22221751.2020.1763209

Conflict of Interest: The authors declare that the research was conducted in the absence of any commercial or financial relationships that could be construed as a potential conflict of interest.

Publisher's Note: All claims expressed in this article are solely those of the authors and do not necessarily represent those of their affiliated organizations, or those of the publisher, the editors and the reviewers. Any product that may be evaluated in this article, or claim that may be made by its manufacturer, is not guaranteed or endorsed by the publisher.

Copyright $\odot 2021$ Tian, Wang, Li, Chen, Zhou, Huang, Sheng and Jiang. This is an open-access article distributed under the terms of the Creative Commons Attribution License (CC BY). The use, distribution or reproduction in other forums is permitted, provided the original author(s) and the copyright owner(s) are credited and that the original publication in this journal is cited, in accordance with accepted academic practice. No use, distribution or reproduction is permitted which does not comply with these terms. 